\section{Parentage and Genetic Diversity Studies on Pangolin (Manis javanica) using Novel Microsatellite Markers}

\author{
Hui-Ming Li ${ }^{1}$, Bi-Ze Yang ${ }^{1}$, Xue-Jun Jin ${ }^{1,2}$, Lin-Miao Li ${ }^{1}$, Hai-Ying Jiang', \\ Xiu-Juan Zhang' and Jin-Ping Chen ${ }^{1 *}$ \\ ${ }^{1}$ Guangdong Key Laboratory of Animal Conservation and Resource Utilization, \\ Guangdong Public Laboratory of Wild Animal Conservation and Utilization, \\ Guangdong Institute of Applied Biological Resources, Guangdong Academy of Science, \\ Guangzhou 510260, China \\ ${ }^{2}$ Dongguan Institute of Qingfengyuan Animal Medicine, Dongguan, Guangdong, China
}

\begin{tabular}{l} 
Article Information \\
\hline Received 16 August 2019 \\
Revised 21 October 2019 \\
Accepted 30 October 2019 \\
Available online 11 October 2021 \\
Authors' Contribution \\
HL and JC designed research. HL an- \\
alysed data and wrote the manuscript. \\
BY developed software necessary to \\
perform and record experiments. LL, \\
XZ and HJ provided expertise and \\
advice on computational analysis. XJ \\
supported all the samples. All authors \\
edited the manuscript. \\
Key words \\
Manis javanica, Microsatellites, \\
Wildlife, Paternity testing, Genetic \\
diversity
\end{tabular}

\begin{abstract}
A B S T R A C T
The Malayan pangolin (Manis javanica) is an endangered mammal species exhibiting scales characteristics. The conservation and management of this species could benefit from a better understanding of its genetic diversity and structure. In this study, 24 novel SSRs were isolated from full-length transcriptome and they were used for assessing of parent-offspring relationship for M. javanica. All SSR markers were highly polymorphic with a mean of 6 alleles per locus, ranged from 2 to 10 alleles. The average polymorphism information content (PIC) was 0.62 . The observed $\left(H_{O}\right)$ and expected average heterozygosity $\left(H_{E}\right)$ value was 0.56 and 0.67 tested in 39 individual samples. For parentage testing, the allele frequency date-based indicated that the combined exclusion probability values were over $99.99 \%$ when 9 SSRs were used. This study demonstrated that the microsatellite-based approach could be effectively utilized for parentage analysis in pangolins and that it has a significant application in selective breeding in endangered and valuable mammalian species.
\end{abstract}

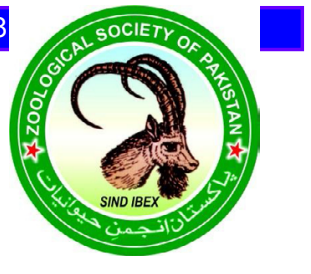

\section{INTRODUCTION}

$\mathrm{P}$ angolins are mammals exhibiting atypical morphological characteristics and belong to Manis, order Pholidota (Gaudin et al., 2006). These mammals have overlapped horny scales on the major parts of the body and a buccal system adapted to a myrmecophagy that makes them unique among the Old World mammalian fauna (Luo et al., 2007). A total of eight extant species of pangolins have been recognized and are widely distributed in Africa and Asia due to the exploitation of natural resources, as well as medicinal demand for pangolin scales and meat (Gaubert and Antunes, 2005). In addition, the illegal trade of pangolin is becoming increasingly serious, and all pangolin species are listed in the Conservation on International Trade in Endangered Species of Wild Fauna and Flora (CITES I) (Kumar et al., 2016; Li et al., 2017).

Although in situ protection is an important component of endangered species protection, in order to expand the existing population and increase genetic diversity, artificial

\footnotetext{
* Corresponding author: chenjp@giabr.gd.cn 0030-9923/2021/0006-2321 \$9.00/0

Copyright 2021 Zoological Society of Pakistan
}

breeding and ex situ conservation have become important measures to protect endangered animals (Wu et al., 2002). Under the conditions of artificial breeding, endangered animals can be guaranteed more comprehensive and adequate food and nutrition and can receive good health protection while avoiding harm of predators $(\mathrm{Wu}$ et al., 2002; Yusoff et al., 2016). Pedigree information can provide relevant data for breeders to make pertinent decisions, since it allows for maintenance of high levels of variability in the progeny, increase of the heterosis effect, and reduction of the levels of inbreeding (Spanoghe et al., 2015; Tarn et al., 1992). Therefore, it is particularly important to establish a stable population of pangolins that is kept artificially in order to avoid inbreeding depression and genetic drift, increase the effective population size and to select and optimize pairing for the purpose of long-term gene exchange between the individuals with the smallest relationship. In this way, not only can the domestic and foreign social needs of scientific research and public education be met, but also certain conditions for the release of captive breeding individuals to supplement wild populations can be created.

Since the 1980s, parentage analysis of animals has been widely expended not only from the development 
of methods for assessing the parentage of individual progeny but also from the increase in the diversity and availability of molecular markers (Luís et al., 2002; Zhang et al., 2016). SSRs (Simple Sequences Repeats) have become the markers of choice in both animal and plant species because of their discriminatory power, codominant transmission, and reproducible properties (Ellegren, 2004; Zietkiewicz et al., 1994). To date, microsatellite-based parentage identification technology is the most widely used and reliable method for the identification of genetic relationships among endangered species, such as Amur tigers (Zhang et al., 2003a), the giant panda (Zhang et al., 2003b), long-snouted seahorse (Hippocampus guttulatus) (Pardo et al., 2007), Shinisaurus crocodilurus (Luo, 2014), chimpanzees (Zhang et al., 2000), Alligator mississippiensis (Davis et al., 2001), Dermochelys coriacea (Crim et al., 2002), Acipenser sturio (Roques et al., 2016) and Falco cherrug (Hou et al., 2018). Moreover, microsatellite analysis only requires a small amount of DNA when sampling endangered and rare species by using nondestructive methods, which can be effectively analyzed and facilitated in field investigation.

In China, the Guangdong province wildlife rescue center has rescued some pangolins from the illegal trad of wild animals. These individuals were raised in Dongguan Institute of Qingfengyuan Animal Medicine; however, the source and relationship between members has not been thoroughly elucidated to date. To restore this rescued population, we combined the nondestructive extraction DNA method and the microsatellite sequences in a known transcriptome of M. javanica (Ma et al., 2019) to carry out new primers and polymorphic gene loci and to establish a highly sensitive paternity identification method for one breeding population of $M$. javanica.

\section{MATERIALS AND METHODS}

One natural death M. javanica and 39 saliva samples (23 males and 16 females) of breeding pangolins were provided by the Dongguan Institute of Qingfengyuan Animal Medicine in 2018. Samples were stored in RNAiso reagent (Takara, Otsu, Japan) and treated with DNase I (Takara). The total RNA(Ma et al., 2019) for transcriptome sequencing and locating microsatellite loci. Saliva samples were stored in $95 \%$ ethanol and kept at $4{ }^{\circ} \mathrm{C}$. From the pedigree record, we know that the parents of "BB" (ㅇ) were "A26" ( $\left(\right.$ P) and "A25" $\left(\mathrm{O}^{\Uparrow}\right)$ in this sample population.

The genomic DNA was isolated using a HiPure Tissue DNA mini kit (Magen Inc.). Randomly selected 30 microsatellite primers for PCR amplification to screen primers that preliminarily possessed polymorphic loci. The polymerase chain reactions (PCR) were conducted in a $10 \mu \mathrm{L}$ reaction volume containing $5 \mu \mathrm{L} 2 \times$ EasyTaq PCR SuperMix (Trangen Inc.), $1 \mu \mathrm{L}$ FAM-labeled M13 forward primer, $0.4 \mu \mathrm{L}$ unlabeled M13-tailed forward primer and $1 \mu \mathrm{L}$ reverse primer, as well as 50-100 ng of genomic DNA template in $1 \mu \mathrm{L}$ (Boutin-Ganache et al., 2001). PCR was performed with the following protocols: one cycle of denaturation at $95{ }^{\circ} \mathrm{C}$ for $10 \mathrm{~min} ; 35$ cycles of $95^{\circ} \mathrm{C}$ for 15 $\mathrm{s}$ with an annealing temperature $52^{\circ} \mathrm{C}$ for $30 \mathrm{~s}$, extension at $72^{\circ} \mathrm{C}$ for $30 \mathrm{~s}$, and then a final extension at $72^{\circ} \mathrm{C}$ for 30 min. PCR products were analyzed with an ABI 3730XL Genetic Analyzer (Applied Biosystems). Fragment sizes were determined by comparison with the internal standard using GeneScan 500-ROX (Applied Biosystems) and GeneMarker version 1.5 (SoftGenetics, State College, Pennsylvania).

The genetic diversity parameters of SSR loci, including the number of alleles $(\mathrm{Na})$, observed heterozygosity $(\mathrm{Ho})$, expected heterozygosity $(\mathrm{He})$, alleles range and polymorphism information content (PIC), were calculated using the software CERVUS 3.0 (Kalinowski et al., 2007). Deviation from Hardy-Weinberg equilibrium $(H W E)$ and linkage disequilibrium (LD) were performed using the software POPGENE V1.31 (Yeh et al., 2000) and FSTAT V2.9.4 (http://www.unil.ch/izea/softwares/ fstat). For paternity testing, the exclusion probabilities of each locus based on the genotype of no known parent and on the genotype of one known parent were named NE-1P, NE-2P and NE-PP, respectively. In addition, the combined exclusion probability over the 24 loci were also calculated.

To estimate the feasibility and confidence of parentage analysis, a simulation program was designed with the software CERVUS 3.0 using the following parameters: parent pair (sex known), 70 candidate parents, 200 offspring, $30 \%$ of the candidate parents were sampled, and proportion of mistyped loci was $1 \%$. In the program, the most-likely parents of each individual were assigned a statistic delta score and LOD score, and the true parents were evaluated depending on the scores of the candidate parents. In addition, a double-blind test was performed to evaluate the capacity of these microsatellite markers.

\section{RESULTS}

Identification and genetic diversity of microsatellite markers According to the full-length transcriptome of $M$. javanica, a total 18,693 SSR sites were detected, including 12,120 mononucleotide repeat sequences, 3,202 dinucleotide repeat sequences, 1,594 tri-nucleotide repeat sequences, 259 tetra-nucleotide repeat sequences, 52 penta-nucleotide repeat sequences and 23 hexa-nucleotide repeat sequences. According to the obtained SSR sites, 56,079 pairs of primers were designed by the software Primer Primer 5.0. 
Table I. Characterization of $\mathbf{2 4}$ microsatellites loci and conditions of PCR in Malayan pangolin.

\begin{tabular}{|c|c|c|c|c|c|c|}
\hline Primers & Accession no. & Repeat type & FPr1(5'-3') / RPr1(5'-3') & Temperature $\left({ }^{\circ} \mathrm{C}\right)$ & PSize (bp) & SSR locus \\
\hline \multirow[t]{2}{*}{ M13MJ-F2 } & MK121608 & (TCCA)9 & TTTCATACCGGGAAGTCCAC & 59.8 & 169 & PB676 \\
\hline & & & ATGGTCCTAACACCACGGAG & 59.8 & & \\
\hline \multirow[t]{2}{*}{ M13MJ-F3 } & MK121609 & (TTTA) 8 & CACCTGCATGTACCCCTTTT & 59.9 & 226 & PB210 \\
\hline & & & СССССТСААААТАССАССТТ & 60.0 & & \\
\hline \multirow[t]{2}{*}{ M13MJ-F4 } & MK121610 & $(\mathrm{ATTC}) 8$ & GAGAGAAAGGGGAAAATCGG & 60.0 & 237 & PB220 \\
\hline & & & TGATAGGATGTGAGGAGGGG & 59.9 & & \\
\hline \multirow[t]{2}{*}{ M13MJ-F5 } & MK121611 & $(\mathrm{AATT}) 8$ & TGGGGTCTGCTGTTTTCATT & 60.5 & 194 & PB235 \\
\hline & & & СTCCCTCTGTAGGTTGCCCT & 60.6 & & \\
\hline \multirow[t]{2}{*}{ M13MJ-F7 } & MK121612 & $(\mathrm{TCAT}) 13$ & AGAAGTGATTTGCACCCCTG & 60.1 & 228 & PB272 \\
\hline & & & CAGTGGCCAGAATGGAGATT & 60.1 & & \\
\hline \multirow[t]{2}{*}{ M13MJ-F9 } & MK121614 & $(\mathrm{ATGA}) 8$ & CCCTATGAGGTGGGCACTAA & 59.9 & 276 & PB387 \\
\hline & & & AACTCCATCAAAGGTGTGGC & 60.0 & & \\
\hline \multirow[t]{2}{*}{ M13MJ-F10 } & MK121615 & $($ GAAT) 8 & CCCAGATCCAAAATGAATGG & 60.1 & 209 & PB442 \\
\hline & & & TGCTGATGTTCACTCTTGCC & 60.0 & & \\
\hline \multirow[t]{2}{*}{ M13MJ-F11 } & MK121616 & (GATA) 12 & ATCCACCTAGGAACCTCAGC & 58.2 & 249 & PB464 \\
\hline & & & GACTCTTCGGGATTTCACACA & 60.1 & & \\
\hline \multirow[t]{2}{*}{ M13MJ-F12 } & MK121617 & (GATA) 12 & ATCCACCTAGGAACCTCAGC & 58.2 & 242 & PB4641 \\
\hline & & & GACTCTTCGGGATTTCACACA & 60.1 & & \\
\hline \multirow[t]{2}{*}{ M13MJ-F17 } & MK121619 & (ATAG) 12 & GTAATGGGGTATGTGGTGGG & 59.8 & 213 & PB537 \\
\hline & & & TCCCTGTTCAAACGGAATTT & 59.4 & & \\
\hline \multirow[t]{2}{*}{ M13MJ-F20 } & MK121620 & $($ AGAT) 16 & CAGTGCTCATCACATAGCAGG & 59.5 & 177 & PB652 \\
\hline & & & CATGCCTAGTGTTTCACGTTG & 59.3 & & \\
\hline \multirow[t]{2}{*}{ M13MJ-F24 } & MK121621 & (TTTTG)7 & TTCAGCCAGGGTCTCTCAGT & 60.0 & 188 & PB714 \\
\hline & & & TGGGGTTTTTCCTCAATCTG & 59.9 & & \\
\hline \multirow[t]{2}{*}{ M13MJ-F25 } & MK121622 & $(\mathrm{AAACA}) 9$ & CCAGAGAAAGGTAGGAGCCA & 59.4 & 128 & PB383 \\
\hline & & & TCCAGAAAACAGACCCAAGG & 60.1 & & \\
\hline \multirow[t]{2}{*}{ M13MJ-F40 } & MK896876 & $(\mathrm{AG}) 10$ & GCAGCAGAACTCATCCAACA & 60.0 & 191 & PB221 \\
\hline & & & TACAGGGAATGGTTTCAGCC & 60.0 & & \\
\hline \multirow[t]{2}{*}{ M13MJ-F41 } & MK896877 & $(\mathrm{AT}) 7$ & GCTCTGAGAATTGCCCATGT & 60.2 & 194 & PB251 \\
\hline & & & CACTGACGAGCAAAGCCATA & 60.0 & & \\
\hline \multirow[t]{2}{*}{ M13MJ-F43 } & MK896879 & $(\mathrm{AT}) 8$ & TTGTCCTGGGGATCTTAGAAAA & 59.9 & 186 & PB681 \\
\hline & & & AATGGGCATTGTCTCCAAAA & 60.3 & & \\
\hline \multirow[t]{2}{*}{ M13MJ-F44 } & MK896880 & $(\mathrm{AC}) 12$ & GGTTGGGGAGGGTGTAACTT & 60.0 & 269 & PB821 \\
\hline & & & TACTCCTTGGAGGCGTGACT & 59.9 & & \\
\hline \multirow[t]{2}{*}{ M13MJ-F47 } & MK896881 & (TC) 10 & AAATCTGTGGCCATCTCTCC & 59.1 & 251 & PB169 \\
\hline & & & GCCTTCCAGGTTGTTCAGAG & 59.8 & & \\
\hline \multirow[t]{2}{*}{ M13MJ-F48 } & MK896882 & $(\mathrm{AC}) 9$ & AGAGCTGGGGAGGATGATTC & 60.6 & 267 & PB171 \\
\hline & & & ACCCTGCAGGAGTCTGAATG & 60.3 & & \\
\hline \multirow[t]{2}{*}{ M13MJ-F49 } & MK896883 & $(\mathrm{TA}) 7$ & TCCCATCTAGAGAGCCATGC & 60.3 & 160 & PB1982 \\
\hline & & & ACCCAGGGTGTGTACCCATA & 60.0 & & \\
\hline \multirow[t]{2}{*}{ M13MJ-F50 } & MK896884 & (TG) 10 & GGGCTATTAATTCTGCCAGC & 58.8 & 264 & PB1983 \\
\hline & & & GTGCATGTTCACACACACATC & 58.4 & & \\
\hline M13MJ-F53 & MK896885 & (TG)8 & ATGTCCTCATCAGGCAAACC & 60.0 & 231 & PB236 \\
\hline & & & CCTAGACTTACCTGCTTGAGCC & 60.0 & & \\
\hline M13MJ-F54 & MK896886 & $(\mathrm{AT}) 6$ & GAGGGCCTTTAGGAAACTGC & 60.2 & 197 & PB2365 \\
\hline & & & GCTTCTAACAATTCCAGAGCTAAA & 58.3 & & \\
\hline M13MJ-F55 & MK896887 & $(\mathrm{AT}) 6$ & GAGGGCCTTTAGGAAACTGC & 60.2 & 198 & PB2366 \\
\hline & & & GCTTCTAACAATTCCAGAGCTAAA & 58.4 & & \\
\hline
\end{tabular}


Of the 30 primer pairs tested, $24(80 \%)$ produced a clear product following amplification of samples for M. javanica. Thirty-nine Malayan pangolins showed a relatively high level of genetic variability (Supplementary Table I), and all 24 loci were polymorphic (Table I), of which 10 loci contained at least 6 alleles. The number of alleles $(\mathrm{Na})$ per locus varied from 2 to 10 , the observed heterozygosity $(\mathrm{Ho})$ in Malayan pangolin ranged from 0.167 to 0.795 , the expected heterozygosity $(\mathrm{He})$ ranged from 0.216 to 0.853 , and the polymorphism information content (PIC) ranged from 0.202 to 0.853 . The means of $\mathrm{Na}, \mathrm{Ho}, \mathrm{He}$ and PIC were 5.5, 0.558, 0.673 and 0.617, respectively. The Hardy-Weinberg test results indicated that 22 markers followed Hardy-Weinberg equilibrium. No significant pair-wise linkage disequilibrium was found between loci after Bonferroni correction $(P<0.000181)$ (Table II, Supplementary Table II).

Table II. Genetic diversity of 24 microsatellite markers in 35 Manis javanica individuals.

\begin{tabular}{|c|c|c|c|c|c|c|c|c|c|}
\hline Locus & $\mathrm{k}$ & $\mathrm{Na}$ & Ho & $\mathrm{He}$ & PIC & NE-1P & NE-2P & NE-PP & HW \\
\hline PB676 & 6 & 39 & 0.641 & 0.701 & 0.638 & .723 & 0.557 & 0.377 & NS \\
\hline PB210 & 6 & 38 & 0.632 & 0.746 & 0.692 & 0.672 & 0.497 & 0.314 & NS \\
\hline PB220 & 5 & 39 & 0.436 & 0.657 & 0.605 & 0.759 & 0.586 & 0.399 & NS \\
\hline PB235 & 7 & 39 & 0.385 & 0.635 & 0.584 & 0.772 & 0.601 & 0.411 & NS \\
\hline PB272 & 5 & 33 & 0.727 & 0.763 & 0.711 & 0.652 & 0.474 & 0.291 & NS \\
\hline PB387 & 5 & 38 & 0.474 & 0.511 & 0.477 & 0.859 & 0.692 & 0.509 & NS \\
\hline PB442 & 8 & 39 & 0.795 & 0.8 & 0.762 & 0.579 & 0.4 & 0.213 & NS \\
\hline PB464 & 6 & 37 & 0.568 & 0.796 & 0.754 & 0.598 & 0.418 & 0.235 & NS \\
\hline PB4641 & 6 & 37 & 0.459 & 0.749 & 0.704 & 0.658 & 0.477 & 0.285 & NS \\
\hline PB537 & 10 & 38 & 0.632 & 0.847 & 0.816 & 0.492 & 0.323 & 0.148 & NS \\
\hline PB652 & 6 & 35 & 0.6 & 0.802 & 0.759 & 0.59 & 0.412 & 0.23 & NS \\
\hline PB714 & 5 & 36 & 0.583 & 0.727 & 0.673 & 0.696 & 0.52 & 0.336 & NS \\
\hline PB383 & 5 & 36 & 0.722 & 0.737 & 0.678 & 0.691 & 0.518 & 0.339 & NS \\
\hline PB221 & 5 & 32 & 0.406 & 0.573 & 0.495 & 0.832 & 0.696 & 0.54 & NS \\
\hline PB251 & 4 & 37 & 0.757 & 0.697 & 0.629 & 0.741 & 0.578 & 0.409 & NS \\
\hline PB681 & 10 & 36 & 0.5 & 0.853 & 0.822 & 0.483 & 0.316 & 0.144 & ND \\
\hline PB821 & 4 & 32 & 0.5 & 0.708 & 0.637 & 0.735 & 0.572 & 0.405 & NS \\
\hline PB169 & 3 & 30 & 0.167 & 0.216 & 0.202 & 0.977 & 0.891 & 0.804 & ND \\
\hline PB171 & 2 & 31 & 0.419 & 0.508 & 0.375 & 0.875 & 0.813 & 0.719 & NS \\
\hline PB1982 & 4 & 33 & 0.727 & 0.597 & 0.533 & 0.815 & 0.66 & 0.491 & NS \\
\hline PB1983 & 3 & 32 & 0.75 & 0.538 & 0.419 & 0.86 & 0.775 & 0.661 & NS \\
\hline PB236 & 6 & 29 & 0.655 & 0.736 & 0.689 & 0.673 & 0.491 & 0.295 & NS \\
\hline PB2365 & 5 & 30 & 0.333 & 0.568 & 0.498 & 0.837 & 0.693 & 0.534 & NS \\
\hline PB2366 & 5 & 29 & 0.517 & 0.7 & 0.635 & 0.732 & 0.564 & 0.386 & NS \\
\hline
\end{tabular}

$\mathrm{Na}$ the number of alleles, $\mathrm{Ho}$ observed heterozygosity, $\mathrm{He}$ expected heterozygosity, PIC polymorphic information content, $* *$ or * Indicated significant deviation from HW after Bonferroni correction $(p<0.005$ or $p<0.01)$.

\section{Parentage analysis}

The exclusion probability of individual locus ranged from 0.144 to 0.804 (Table II) when both parents information were not known (NE-PP), and its combined exclusion probability values were higher than $99 \%$ and 99.99\% when 4 and 9 SSRs were used separately (Fig. 1); The exclusion probability of individual locus ranged from 0.316 to 0.891 (Table II) when one parent information was known (NE-2P), and its combined exclusion probability values were higher than $99 \%$ and $99.99 \%$ when 3 and 5 SSRs were used separately (Fig. 1); The exclusion probability of individual locus ranged from 0.483 to 0.977 (Table II) when information for both parents were known (NE-1P), and its combined exclusion probability values was higher than $99.99 \%$ when 3 SSRs were used (Fig. 1). This test confirms that the parents of "BB" (P) were "A26" (ㅇ) and "A25" ( $\left.{ }^{\Uparrow}\right)$, which was consistent with the pedigree record from Qingfengyuan medicine institute. In addition, the results also supported that "C9" and "72" were parent-offspring relationships (Supplementary Table III).

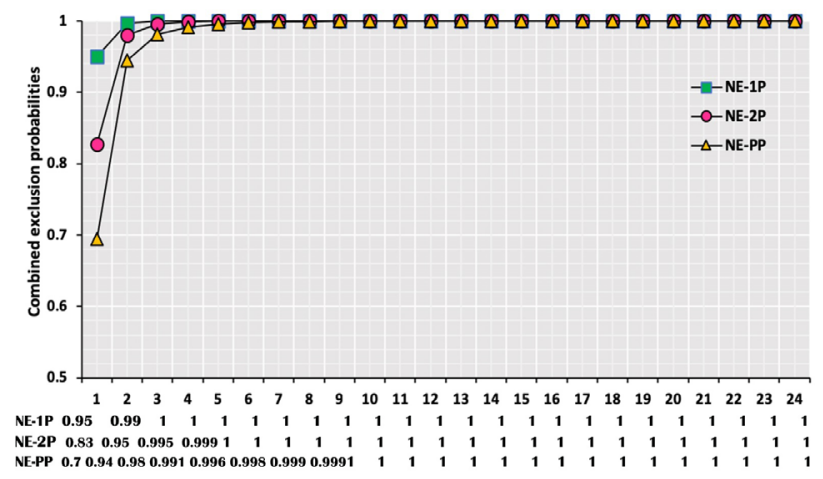

Fig. 1. Combined exclusion probabilities of 24 polymorphic loci analyzed for parent known (E-1P) and one parent known (E-2P).

\section{DISCUSSION}

In this study, we firstly used 24 newly isolated SSR loci to identify the paternity of a breeding population of M. javanica. Although Luo et al. (2007) had screened 32 polymorphic microsatellite markers for M.javanica in 2007, theusefulnessoftheselociforpaternity testing wasnotfurther validated due to the rarity of species and fewer samples.

The level of population genetic diversity directly reflects the individual's ability to adapt to the environment. The greater the abundance in variation of genetic diversity within a species is, the greater the adaptability of the species to environmental changes is (Michaux et al., 2005). In our study, the genetic polymorphism of the artificially raised $M$. javanica population was relatively high, 20 loci of $24 \mathrm{SSRs}$ 
showed high polymorphism (PIC $>0.5$ ), and the average observed heterozygosity $(\mathrm{Ho})$ and expected heterozygosity $(\mathrm{He})$ in Malayan pangolin were 0.557 and 0.673 , respectively. This finding indicated that the population of artificially raised pangolin can avoid inbreeding under correct guidance, which is conductive to the recovery of the pangolin population (Botstein et al., 1980).

Compared with $\mathrm{He}$ of other rare and protected animals, such as the giant panda $(H e=0.637)$ (Zhang et al., 2003b), American alligator $(\mathrm{He}=0.76)$ (Davis et al., 2001), Chinese crocodile $(H e=0.54)$ (Luo, 2014), Rhesus macaques $(H e=0.62)$ (Morin et al., 1997) and Lynx (He $=0.53$ ) (Carmichael et al., 2000), the genetic diversity of $M$. javanica was not significantly higher than that of other species. The low genetic diversity in $M$. javanica observed in this preliminary microsatellite survey suggests an urgent need for population management and conservation of this species, since the loss of genetic diversity in endangered species is often associated with inbreeding and a reduction in reproductive fitness (Reed and Frankham, 2001).

In our study, the combined exclusion probability for NE-1P and NE-2P were higher than $99.99 \%$ when 4 SSRs were used. Moreover, when 9 SSRs were used, the values of three type parents information was higher than $99.99 \%$. In previous reports on paternity testing, usually 6 SSRs markers may make the exclusion probability of pedigree confirmation more than 99\% (Zajc, 1999). 10 and 18 polymorphic SSR loci can be used to accurately identify the relationship between individuals of Amur tiger (Zhang et al., 2003a) and Acipenser sturio (Roques et al., 2016) separately. These results indicated that the selected SSRs had a very high power and were suitable for parentage analysis. The more loci that we selected, the higher the accuracy was that could be reached in parentage analysis.

In conclusion, the parentage analysis investigated in this study, which was based on kinship testing and inferential methods, was demonstrated to be particularly hopeful regarding the development of pedigree information validation or parental assignment programs. As a genetic marker, microsatellites can objectively reflect the genetic diversity of pangolin and can be used in a paternity test. Therefore, the combination of microsatellite markers and traditional ecological research for carrying out genetic diversity analysis and the paternity test of pangolin will promote the protection, as well as rational development and utilization, of rare species resources and scientific resource management.

\section{ACKNOWLEDGEMENTS}

This study was supported by one support program: the Certificate of China Postdoctoral Science Foundation
Grant (2019M652832), the GDAS' Project of Science and Technology Development (2019GDASYL-0103062), the Guangdong Institute of Applied Biological Resources postdoctoral training fund (GIABR-pyjj2018020), and the GDAS Special of Science and Technology Development (2018GDASCX-0107).

\section{Supplementary material}

There is supplementary material associated with this article. Access the material online at: https://dx.doi. org/10.17582/journal.pjz/20190816080848

\section{Statement of conflict of interest}

The authors have declared no conflict of interest.

\section{REFERENCES}

Botstein, D., White, R.L., Skolnick, M. and Davis, R.W., 1980. Construction of a genetic linkage map in man using restriction fragment length polymorphisms. Am. J. Hum. Genet., 32: 314-331.

Boutin-Ganache, I., Raposo, M., Raymond, M. and Deschepper, C.F., 2011. M13-tailed primers improve the readability and usability of microsatellite analyses performed with two different allele-sizing methods. Biotechniques, 31: 24-26.

Carmichael, L.E., Clark, W. and Strobeck, C., 2000. Development and characterization of microsatellite loci from lynx (Lynx canadensis), and their use in other felids. Mol. Ecol., 9: 2197-2199. https://doi. org/10.1046/j.1365-294X.2000.105323.x

Crim, J.L., Spotila, L.D., Spotila, J.R., Reina, M.R., Williams, C.J. and Palanino, F.V., 2002. The leatherback turtle, Dermochelys coriacea, exhibits both polyandry and polygyny. Mol. Ecol., 11: 2097-2106. https://doi.org/10.1046/j.1365294X.2002.01591.x

Davis, L.M., Glenn, T.C., Elsey, R.M., Dessauers, H.C. and Sawyer, R.H., 2001. Multiple paternity and mating patterns in the American alligator, Alligator mississippiensis. Mol. Ecol., 10: 1011-1024. https://doi.org/10.1046/j.1365-294X.2001.01241.x

Ellegren, H., 2004. Microsatellites: Simple sequences with complex evolution. Nat. Rev. Genet., 5:435445. https://doi.org/10.1038/nrg1348

Gaubert, P. and Antunes, A., 2005. Assessing the taxonomic status of the Palawan pangolin Manis culionensis (Pholidota) using discretemorphological characters. J. Mammal., 86: 1068-1074. https://doi. org/10.1644/1545-1542(2005)86[1068:ATTSOT]2 $.0 . \mathrm{CO} ; 2$

Gaudin, T.J., Emry, R.J. and Pogue, B., 2006. A new 
genus and species of pangolin (Mammalia, Pholidota) from the late Eocene of Inner Mongolia, China. J. Vertebr. Paleontol., 26: 146-159. https:// doi.org/10.1671/0272-4634(2006)26[146:ANGAS $\mathrm{O}] 2.0 . \mathrm{CO} ; 2$

Hou, X., Xu, P., Lin, Z., D’Urban-Jackson, J., Dixon, A., Bold, B., Xu, J. and Zhan, X., 2018. An integrated tool for microsatellite isolation and validation from the reference genome and their application in the study of breeding turnover in an endangered avian population. Integr. Zool., 13: 553-568. https://doi. org/10.1111/1749-4877.12305

Kalinowski, S.T., Taper, M.L. and Marshall, T.C., 2007. Revising how the computer program CERVUS accommodates genotyping error increases success in paternity assignment. Mol. Ecol., 16: 1099-1106. https://doi.org/10.1111/j.1365-294X.2007.03089.x

Kumar, V.P., Rajpoot, A., Mukesh, Shukla, M., Kumar, D. and Goyal, S.P., 2016. Illegal trade of Indian Pangolin (Manis crassicaudata): Genetic study from scales based on mitochondrial genes. Egypt J. Forensic Sci., 6: 524-533. https://doi.org/10.1016/j. ejfs.2016.06.008

Li, L., Wei, L., Ma, J. and Chen, J., 2017. Development and characterization of 21 SNP markers in Manis javanica based on high-throughput sequencing. Conserv. Genet. Resour., 3: 1-3. https://doi. org/10.1007/s12686-017-0822-1

Luís, C., Cothran, E.G. and Oom, M.M., 2002. Microsatellites in Portuguese autochthonous horse breeds : usefulness for parentage testing. Genet. Mol. Biol., 25: 131-134. https://doi.org/10.1590/ S1415-47572002000200003

Luo, D., 2014. Parentage and genetic diversity studies in Luokeng Chinese crocodile (Shinisaurus crocodilurus) using microsatellite DNA marker. $\mathrm{PhD}$ thesis, Guangxi Normal University, Guilin city, China.

Luo, S.J., Cai, Q.X., David, V.A., Zhang, L., Martelli, P.L., Norman, T.L., Ferrand, N.C., Gauber, S.C., Ramos, P., O’Brien, W.E., Antunes, S.J., Johnosn, A. and Warren, E., 2007. Isolation and characterization of microsatellite markers in pangolins (Mammalia, Pholidota, Manis spp.): Primer note. Mol. Ecol. Notes, 7:269-272. https://doi.org/10.1111/j.14718286.2006.01577.x

Ma, J.E., Jiang, H.Y., Li, L.M., Zhang, X.J., Li, H.M., Li, G.Y., Mo, D.Y. and Chen, J.P., 2019. SMRT sequencing of the full-length transcriptome of the Sunda pangolin (Manis javanica). Gene, 692:208216. https://doi.org/10.1016/j.gene.2019.01.008

Michaux, J., Hardy, O., Justy, F., Fournier, P., Kanz, A.,
Cabria, M., Davison, A., Rosoux, R. and Libois, R., 2005. Conservation genetics and population history of the threatened European mink Mustela lutreola, with an emphasis on the west European population. Mol. Ecol., 14: 2373-2388. https://doi.org/10.1111/ j.1365-294X.2005.02597.x

Morin, P., Kanthaswamy, S. and Smith, D.G., 1997. Simple sequence repeat (SSR) polymorphisms for colony management and population genetics in rhesus macaques (Macaca mulatta). Am. J. Primatol., 42: 199-213. https://doi.org/10.1002/ (SICI) 1098 -2345(1997)42:3<199::AIDAJP3>3.0.CO;2-S

Pardo, B.G., López, A., Martínez, P. and Bouza, C., 2007. Novel microsatellite loci in the threatened European long-snouted seahorse (Hippocampus guttulatus) for genetic diversity and parentage analysis. Conserv. Genet., 8: 1243-1245. https:// doi.org/10.1007/s10592-006-9241-7

Reed, D.H. and Frankham, R., 2001. How closely correlated are molecular and quantitative measures of genetic variation? A metaanalysis. Evolution, 55: 1095-1103. https://doi. org/10.1111/j.0014-3820.2001.tb00629.x

Roques, S., Berrebi, P., Rochard, E. and Acolas, M., 2016. Parentage assignment in the critically endangered European sturgeon (Acipenser sturio) based on a novel microsatellite multiplex assay: a valuable resource for restocking, monitoring and conservation programs. Conserv. Gene. Resour., 8: 313-322.

Spanoghe, M., Marique, T., Rivière, J., Lanterbecq, D. and Gadenne, M., 2015. Investigation and development of potato parentage analysis methods using multiplexed SSR fingerprinting. Potato Res., 58: 43-65. https://doi.org/10.1007/s11540-0149271-3

Tarn, T.R., Ta,i G.C.C., De long, H., Murphy, A.M. and Seabrook, E.A., 1992. Breeding potatoes for longday, temperate climates. Pl. Breed Rev., 9: 217 332. https://doi.org/10.1002/9780470650363.ch8

Wu, S., Ma, G.Z., Tang, M., Chen, M. and Liu, N., 2002. The status and conservation strategy of pangolin resource in China. J. Nat. Resour., 17: 174-180.

Yeh, F., Yang, R., Boyle, T. and Boyle, T., 2000. POPGENE32: Microsoft Windows-based freeware for population genetic analysis. University of Alberta, Edmonton. pp. 32.

Yusoff, A.M., Tan, T.K., Hari ,R., Koepfli, K., Wee, W.Y., Antunes, A., Sitam, F.T., Rovie-Ryan, J.J., Karuppannan, K.V., Wong, G.J., Lipovich, L., Warren, W.C., O'Brien, S.J. and Choo, 
S.W., 2016. De novo sequencing, assembly and anlysis of eight different transcriptomes from the Malayan pangolin. Sci. Rep., 6: 28199. https://doi. org/10.1038/srep28199

Zajc, I., 1999. Utility of canine microsatellite in revealing the relationship of pure bred dogs. J. Hered., 90: 104-107. https://doi.org/10.1093/jhered/90.1.104

Zhang, J., Ma, W., Wang, W., Gui, J. and Mei, J., 2016. Parentage determination of yellow catfish (Pelteobagrus fulvidraco) based on microsatellite DNA markers. Aquac. Int., 24: 567-576. https:// doi.org/10.1007/s10499-015-9947-y

Zhang, Y., Lawrance, S.K., Ryder, O.A., Zhang, Y. and Isaza, R., 2000. Identification of monozygotic chimpanzees by microsatellite analysis. Am. J. Primatol., 52: 101-106. https://doi.
org/10.1002/1098-2345(200010)52:2<101::AIDAJP4>3.0.CO;2-6

Zhang, Y., Li, D., Rao, L., Xiao, Q. and Liu, D., 2003a. Identification of polymorphic microsatellite DNA loci and paternity testing of Amur tigers. Acta Zool. Sin., 49: 118-123.

Zhang, Z., Shen, F., Sun, S., David, V., Zhang, A., O'Brien, A. and Stephen, J., 2003b. Paternity assignment of giant panda by microsatellite genotyping. Hereditas(Beijing), 25: 504-510.

Zietkiewicz, E., Rafalski, J.A., Consulting, R. and Labuda, D., 1994. Genome fingerprinting by simple sequence repeat (SSR)-Anchored polymerase chain reaction amplification. Genomics, 20: 176-183. https://doi.org/10.1006/geno.1994.1151 